\title{
CRYSTALLINE FERRIMAGNET-SPINGLASS-AMORPHOUS FERRIMAGNET PHASE TRANSITION IN Tb/Fe MULTILAYERS
}

\author{
S. HONDA, T. KIMURA, M. NAWATE and T. KUSUDA \\ Physical Electronics, Faculty of Engineering, Hiroshima University, 1-4-1 Kagamiyama, Higashi-Hiroshima 724, Japan
}

\begin{abstract}
ABSTRACr-The crystallographic structure and the magnetic properties of $\mathrm{Tb} / \mathrm{Fe}$ sputtered multilayers have been studied as a function of the thickness of Fe-layer $\left(\mathrm{d}_{\mathrm{Fe}}\right)$. The crystallographic structure of Fe layer changes from bcc to amorphous at the critical thickness of Fe layer $\left(d_{\mathrm{Fe}}{ }^{\mathrm{C}} \fallingdotseq 17 \AA\right)$ with decreasing $\mathrm{d}_{\mathrm{Fe}}$. In corresponding to the change in the crystallographic phase, the magnetic properties of the saturation magnetization $M_{s}$, the Kerr rotation angle $\theta_{k}$, the Curie temperature $T_{c}$ and the perpendicular coercivity $H_{c}(1)$ change abruptly at the critical thickness $d_{F e}{ }^{c}$, where the Curie temperature drops to below room temperature and the magnetization curve shows the spinglass like or superparamagnetism like nature at room temperature. Below $\mathrm{d}_{\mathrm{Fe}}{ }^{\mathrm{c}}$, with decreasing $\mathrm{d}_{\mathrm{Fe}}$ the Curie temperature $T_{c}$ increseases owing to the exchange interaction between $\mathrm{Fe}$ and $\mathrm{Tb}$ around the layer boundaries and then the saturation magnetization increases again approaching the value of the alloys with the same composition. The perpendicular coercivity also increases steeply with decreasing $d_{F e}$.
\end{abstract}

KEYWORDS: Amorphous Fe, Tb/Fe Multilayer, Spinglass, Superparamagnetism, Sputtered Multilayer, Perpendicular Coercivity, Curie Temperature, Magneto-optical Kerr Rotation

\section{INTRODUCTION}

Compositionally modulated rare-earth/ transition-metal films have been extensively studied in recent years for reasons of scientific interest and of application to magneto-optical recording [1 3]. Sato [2] reported that $\mathrm{Tb} / \mathrm{Fe}$ multilayers have large perpendicular magnetic anisotropy for small layer thickness and suggested that the perpendicular anisotropy comes from the anisotropic distribution of $\mathrm{Tb}-\mathrm{Fe}$ pairs aligned perpendicular to the film plane.

Yoden et. al. [4] studied the crystallographic structure of Dy/Fe multilayers and indicated that $\mathrm{Fe}$ layer changes from crystalline to amorphous at about $20 \AA$ with decreasing $F e$ layer thickness $\left(d_{F e}\right)$ and there the magnetization at room temperature falls off to nearly zero. We have also shown that the crystallographic structure of $\mathrm{Fe}$ layer in evaporated Dy/Fe $[5,6]$ and in sputtered DyFe/Fe multilayers [5] changes from bcc to amorphous via fcc at about $20 \AA$, and the saturation magnetization falls off drastically at this point followed by the increase in the magnetization with decreasing $d_{F e}$. Sellmyer and Shan $[7,8]$ examined intensively the periodicity dependence of magnetic properties of $\mathrm{Dy} / \mathrm{Co}, \mathrm{Dy} / \mathrm{Fe}$ and $\mathrm{Tb} / \mathrm{Fe}$ multilayers, and indicated that a "kink" in the curve of magnetization versus $d_{F e}$ appears in $D y / F e$ and $\mathrm{Tb} / \mathrm{Fe}$ multilayers.
In this paper, we will discuss the magnetic phase transition arised from the crystallographic change in $\mathrm{Tb} / \mathrm{Fe}$ sputtered multilayers.

\section{EXPERIMENTS}

$\mathrm{Tb} / \mathrm{Fe}$ multilayers were prepared by $\mathrm{RF}$ magnetron sputtering at 6 mTorr Ar gas pressure from $\mathrm{Tb}$ and $\mathrm{Fe}$ targets onto glass substrates on a rotatable substrate table. Two type multilayers were prepared;Type I is the sequence of substrate/Tb/Fe/.../Fe/ $/ \mathrm{Tb} / \mathrm{SiO}_{2}$ and Type $\|$ is the sequence of substrate $/ \mathrm{Fe} / \mathrm{Tb} / \cdots \cdot \mathrm{Tb} / \mathrm{Fe} / \mathrm{SiO}_{2}$. Here, $\mathrm{SiO}_{2}$ layer of $500 \AA$ thickness was overcoated for the protection. The thickness ratio of $\mathrm{Fe}$ to $\mathrm{Tb}$ layer $\left(\mathrm{d}_{\mathrm{Fe}} / \mathrm{d}_{\mathrm{rb}}\right)$ was held constant $1 / 1,1 / 2,1 / 3$ and $1 / 4$, and the bilayer thickness, $\Lambda=d_{r_{a}}+d_{T_{b}}$, was changed from 6 to $160 \AA$ with the total thickness of about $1800 \AA$. In this paper, we will discuss the results only for the films of $\mathrm{d}_{\mathrm{r}, \mathrm{s}} / \mathrm{d}_{\mathrm{T} \mathrm{b}}=1 / 2$.

The layered structure was confirmed in the range between 20 and $100 \AA$ of periodic length $(\Lambda)$ by the small angle $X$-ray diffraction (SA-XRD). The periodic length $(\Lambda)$ was estimated from the peak position of SA-XRD for $\Lambda \geq 20 \AA$, and calculated for $\Lambda<20 \AA$ from the sputtering rate which was corrected from SA-XRD and the total film thickness. The film composition was determined by Rutherford backscattering spectroscopy (RBS). The magnetic properties 


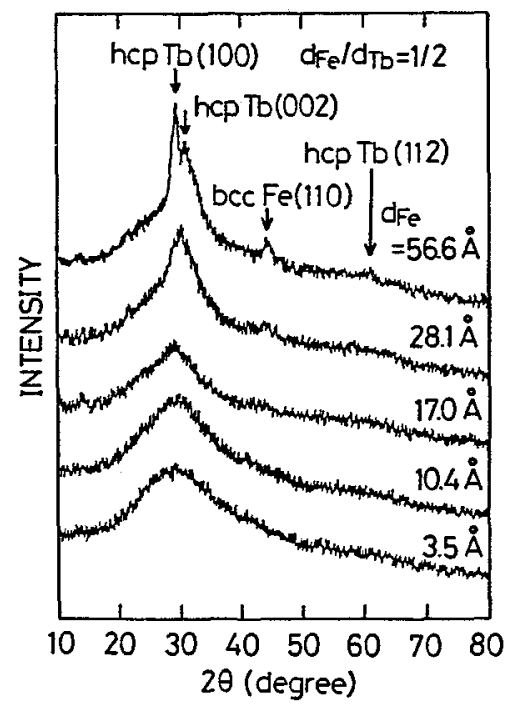

Fig. 1. X-ray diffraction of $\mathrm{Tb} / \mathrm{Fe}$ multilayers of Type Il with various thickness of Fe layer $\left(d_{\mathrm{Fu}}\right)$.
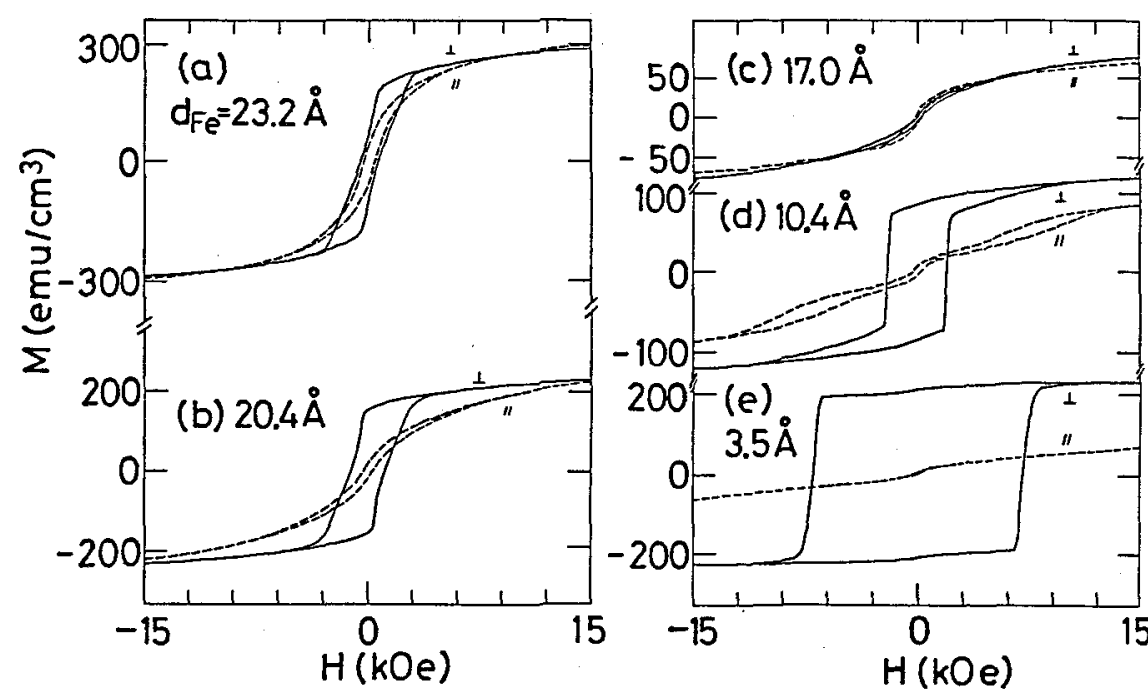

Fig.2. Magnetic hysteresis loops for Type I films with various thickness of Fe layer $\left(d_{\mathrm{Fe}}\right)$. were measured with a vibrating sample magnetometer and a Kerr loop tracer at a wavelength of $780 \mathrm{~nm}$. The crystallographic structure was studied by $X$-ray scattering method.

\section{RESULTS AND DISCUSSION}

X-ray diffraction spectra are shown in Fig. 1 for several modulation periodicities $(\Lambda)$. For $\Lambda=170 \AA \quad\left(d_{\mathrm{Fe}}=56.6 \AA\right.$, $\left.\mathrm{d}_{\mathrm{Tb}}=113.2 \AA\right)$, we can recognize the clear peaks of $(100),(002)$ and (112) planes of hcp $\mathrm{Tb}$ and (110) plane of bcc Fe. These peaks become smaller in intensity and broaden with decreasing $\Lambda$ and only the broad peak due to glass substrate remains around $30^{\circ}$ for $\Lambda \leqq 50 \AA \quad\left(d_{\mathrm{Fe}} \leqq 17 \AA, d_{\text {r b }} \leqq\right.$ $34 \AA$ ) . These data imply that the crystallographic structure changes from crystalline to amorphous at around $17 \AA$ of $d_{F e}$ with decreasing layer thickness similar to the evaporated Dy/Fe and the sputtered DyFe/Fe multilayers $[5,6]$.

The magnetic hysteresis characteristics vary with the Fe layer thickness $\left(d_{F e}\right)$ as shown in Fig.2. Although the easy axis of magnetization lies in film plane for $d_{\mathrm{F}} \geq 40 \AA$, the small loops appear for the perpendicular magnetization curves. The coercivity $\mathrm{H}_{c}(1)$ of the perpendicular loop increases with decreasing $d_{F a}$ and the perpendicular loops become parallelogrammic for $d_{\mathrm{Fe}} \leq 24 \AA$ as shown in Figs.2(a) and (b) , because of the increase in the perpendicular anisotropy originated from the interfacial anisotropy. At the critical layer thickness, $\mathrm{d}_{\mathrm{Fe}} \mathrm{c} \fallingdotseq 17 \AA$, of transition in crystalline phase, the magnetization curves change abruptly and exhibit the spinglass like or the superparamagnetism like nature as shown in Fig.2(c); namely, both the perpendicular and in-plane magnetization curves, which coincide with each other in the experimental error, have not hysteresis characteristic and do not saturate even at $15 \mathrm{kOe}$. Below $\mathrm{d}_{\mathrm{Fe}}{ }^{\mathrm{c}}$, the rectangular hysteresis loops appear again for perpendicular applied field, and the perpendicular anisotropy energy and coercivity increase with decreasing $\mathrm{d}_{\mathrm{Fe}}{ }^{c}$ as shown in Figs.2 (d) and (e).

The similar behaviour with change in $d_{\mathrm{F} e}$ is also observed in polar Kerr loops as shown in Fig.3. In particular, the saturation Kerr rotation angle becomes nearly zero at the critical thickness $d_{F e}{ }^{c}$ (Fig.3c). Below $d_{F e}{ }^{c}$, the polarity of Kerr rotation changes to $\mathrm{Tb}-\mathrm{rich}$ characteristics from Fe-rich ones and rectangular loops with high coercivity appear as shown in Figs.3 (d) and (e) .

Fig. 4 shows the saturation or spontaneous magnetization $M_{s}$ as a function of

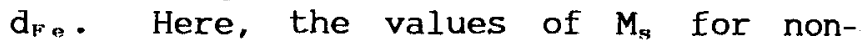
saturated films around $17 \AA$ of $d_{F e}$ are obtained from Arrott plot method and the polarity of $M_{s}$ corresponds to that of negative polar Kerr rotation; namely + or - sign indicates the Fe- or Tb-moment dominant nature. The value of $M_{s}$ decreases gradually with reduction of $d_{F e}$ and becomes zero around $17 \AA$. Below $17 \AA, M_{s}$ is negative, namely $\mathrm{Tb}$-moment is dominant. The absolute value of $M_{s}$ increases again with decreasing $\mathrm{d}_{\mathrm{F}}$ and approaches the value of alloyed amorphous film with the 


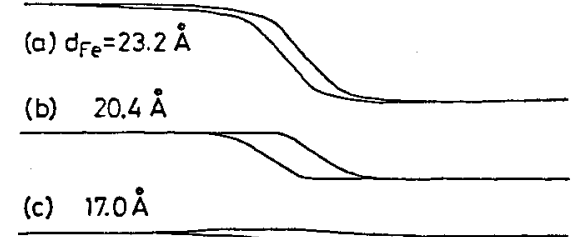

(d) $10.4 \AA$

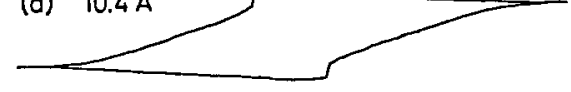

(e) $3.5 \AA$

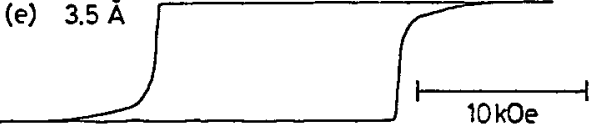

Fig.3. Polar Kerr hysteresis loops measured from the film surface for Type I films of different $\mathrm{d}_{\mathrm{F} \cdot \mathrm{e}}$.

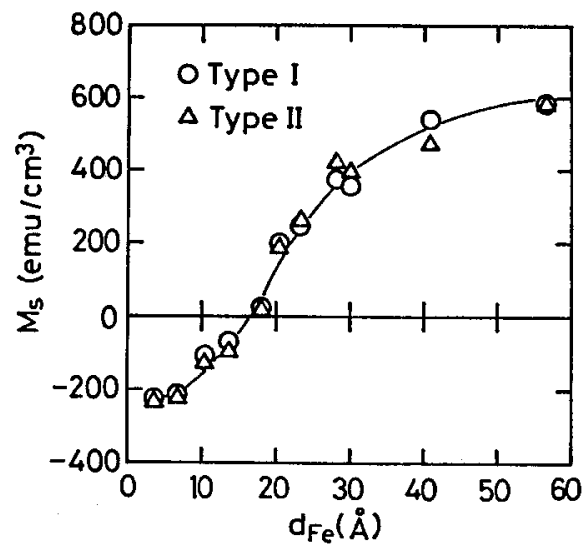

Fig.4. Saturation magnetization $\left(M_{s}\right)$ as a function of $d_{F e}$.

same Tb-composition, 33at.잉 [9] .

The saturation Kerr rotation angle $0_{k}$, which was measured through glass substrate at a wavelength of $780 \mathrm{~nm}$, is plotted in Fig.5. The absolute value of $0_{k}$ is nearly constant $0.35^{\circ}$ in the crystalline region of $d_{\mathrm{Fe}} \geq 20 \AA$ for the films of Type ll, while the value decreases with increasing $d_{1 r}$ above $20 \AA$ for the films of Type 1 . Because the Kerr rotation at $780 \mathrm{~nm}$ wavelength comes mainly from $\mathrm{Fe}$ layer, with increasing $\mathrm{d}_{\mathrm{Fe}}$ or increasing $d_{T}$, the intensity of light reflected from Fe layer decays for Type $\mid$ films and thereby the apparent Kerr rotation angle decreases. The absolute value of $O_{k}$ falls off abruptly to zero at the critical thickness $\mathrm{d}_{\mathrm{F}_{\theta}}$ ", and it increases again gradually with decreasing $\mathrm{d}_{\mathrm{F} \theta}$.

As shown in Fig.6, the perpendicular coercivity $\mathrm{H}_{\mathrm{c}}$ (1) increases slightly up to about 1 kOe with decreasing $d_{k e}$ to $20 \AA$ from $60 \AA$ in the crystalline region. At the critical thickness $\mathrm{d}_{\mathrm{rre}_{\mathbf{e}}}{ }^{\mathrm{c}}, \mathrm{H}_{\mathbf{c}}(1)$ drops to nearly zero and then increases drastically upto about $8 \mathrm{kOe}$ with decreasing $\mathrm{d}_{\mathrm{F} \text { e }}$ in the amorphous region below $d_{\mathrm{Fe}}$ ".

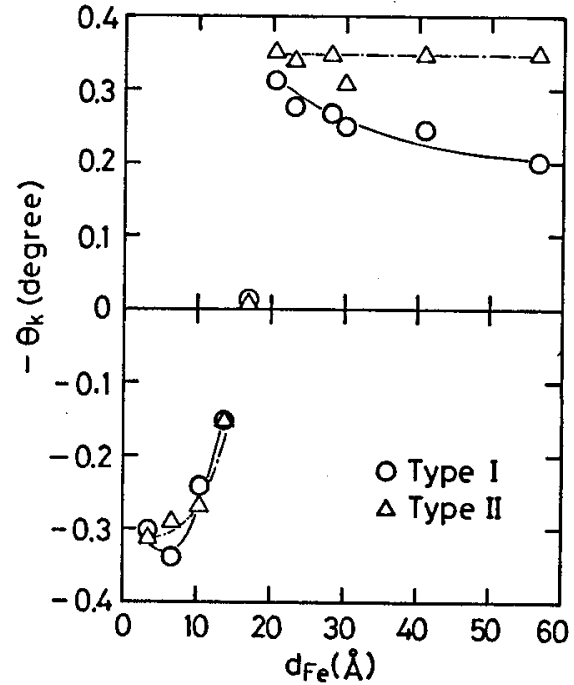

Fig.5. Saturation Kerr rotation angle $\theta_{\mathbf{k}}$ measured through glass substrate at a wavelength of $780 \mathrm{~nm}$.

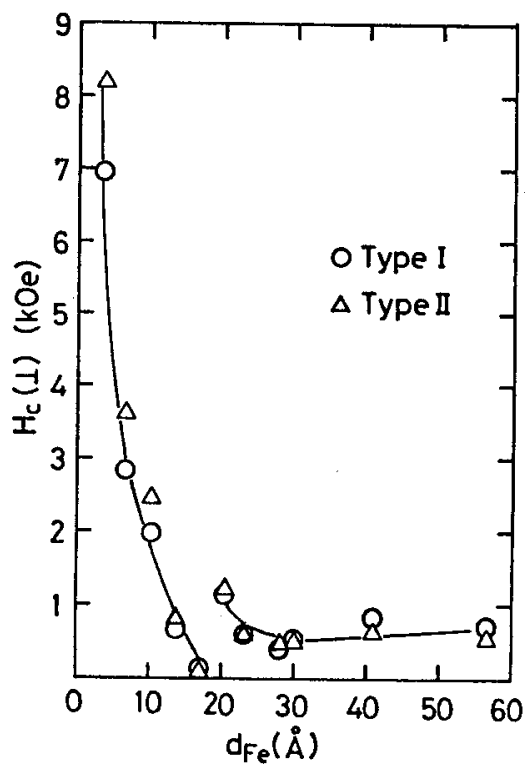

Fig.6. Perpendicular coercivity $H_{c}(1)$ versus $d_{F e}$.

We have seen in the above that the magnetic properties change abruptly at the critical $\mathrm{Fe}$ layer thickness, $\mathrm{d}_{\mathrm{Fe}} \mathrm{c}$, around $17 \AA$ in coincidence with the variation of crystalline structure in $\mathrm{Fe}$ layer. Plausibly, the spinglass like or the superparamagnetism like nature at $\mathrm{d}_{\mathrm{Fe}}{ }^{\mathrm{c}:-17 \AA}$ is closely connected with the amorphous structure of Fe layer, because the Curie temperature of amorphous $\mathrm{Fe}$ is below room temperature as reported in Refs. $[4,10]$.

We, therefore, estimated the Curie temperature $T_{r}$ by measuring the temperature dependence of the Kerr rotation angle $0_{k}$. Temperature dependences of $\left|\theta_{k}\right|$ are plotted in Fig.7 for various $d_{F e}$. For the film of $d_{k e}=20.4 \AA$, the value of $\left|\theta_{k}\right|$ 


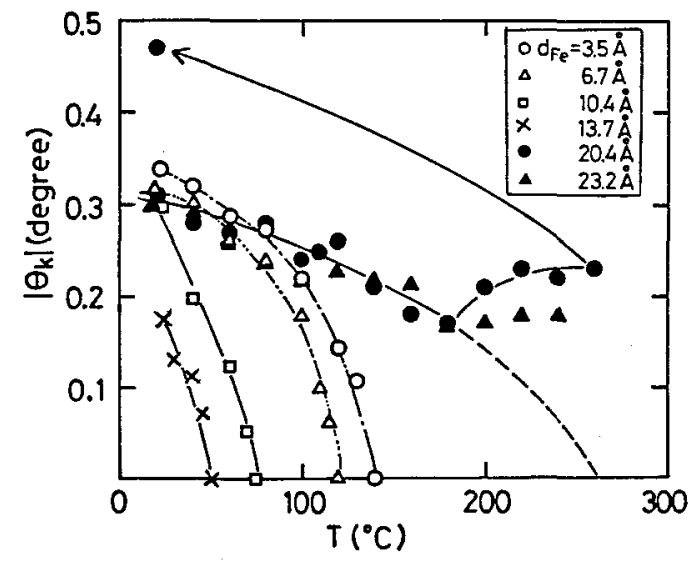

Fig.7. Temperature dependence of $\theta_{k}$ at $780 \mathrm{~nm}$ for the films of Type I.

increases irreversibly with.rising temperature above $200^{\circ} \mathrm{C}$. This irreversible increase is caused by the crystallization of $\mathrm{Fe}$ around the layer boundaries, because the XRD peak of (110) bcc Fe increased after heated up to $300^{\circ} \mathrm{C}$. In this case, the Curie temperature $T_{c}$ was estimated by the extrapolation as shown by the broken line in Fig. 7 .

The Curie temperature $T_{c}$, thereby, is obtained as a function of $d_{p e}$ as shown in Fig.8. We can see that the Curie temperature $T_{c}$ falls off abruptly at around $17 \AA$ with decreasing $\mathrm{d}_{\mathrm{Fe}}$. The abrupt drop in $\mathrm{T}_{\mathrm{c}}$ is probably caused by the phase transition from crystalline to amorphous at this critical layer thickness. This might be supported by the irreversible increase in $\left|\theta_{\mathrm{k}}\right|$ above $200^{\circ} \mathrm{C}$ for the films of $\mathrm{d}_{\mathrm{F} \theta}=$ $20.4 \AA$ as shown in Fig.7. Below the critical $F e$ layer thickness $d_{F e}{ }^{c}$, the Curie temperature rises again owing to the exchange interaction between $\mathrm{Fe}$ and $\mathrm{Tb}$ around the layer boundaries. Then, $\mathrm{Fe}$ moment and especially Tb moment increase, and the net saturation magnetization $\mathbf{M}_{\mathbf{s}}$ increases with the polarity of $\mathrm{Tb}$-dominant nature. The increase in $\mathrm{T}_{\mathrm{c}}$ implies that the Tb atoms become active magnetically with decreasing $d_{F e}$ similarly to the $T_{c}$ change against $\mathrm{Tb}$ composition in amorphous Tb-Fe alloys, in which $\mathrm{T}_{\mathrm{c}}$ rises with increasing $\mathrm{Tb}$ composition [9] .

\section{SUMMARY}

The crystallographic structure of $\mathrm{Fe}$ layer changed from bcc to amorphous at the critical thickness of Fe layer $\left(d_{F_{\theta}}{ }^{c} \fallingdotseq 17 \AA\right)$ with decreasing $\mathrm{d}_{\mathrm{F} \text {. . At the critical }}$ thickness $d_{r_{e}}{ }^{c}$, the curie temperature droped to below room temperature and then the magnetization curve showed the spin-

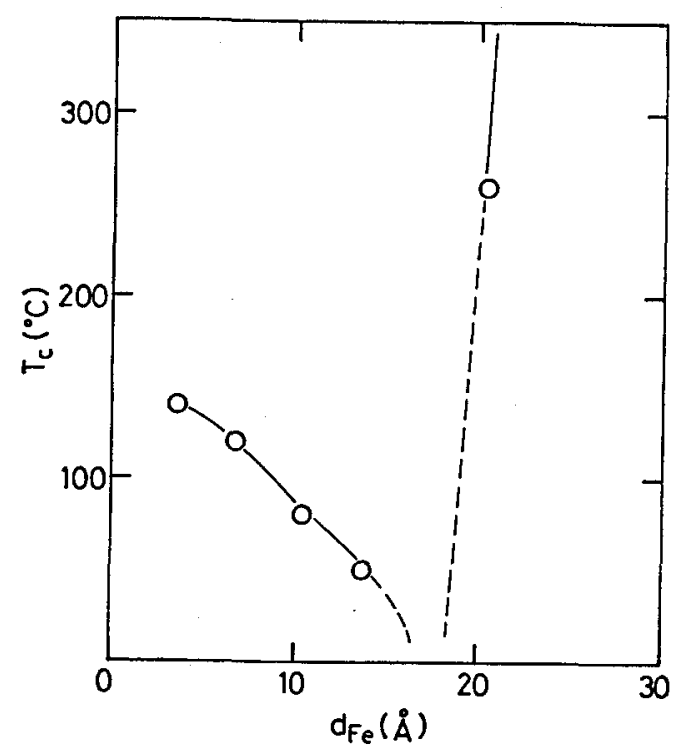

Fig.8. Curie temperature $\mathrm{T}_{c}$ estimated from the temperature dependence of $\theta_{k}$.

glass like or superparamagnetism like nature at room temperature. Below $d_{r_{e}}{ }^{c}$, with decreasing $d_{\mathrm{re}}$ the Curie temperature $\mathrm{T}_{c}$ increseased owing to the exchange interaction between $\mathrm{Fe}$ and $\mathrm{Tb}$ around the layer boundaries, then the saturation magnetization increased again and the perpendicular coercivity increased drastically.

\section{ACKNOWLEDGMENTS}

The authors wish to thank F.Nishiyama for RBS mea-surements. This work was supported by a Grant-in-Aid for Scientific Research on Priority Areas from the Ministry of Education, Science and Culture.

\section{REFERENCES}

[1]T.Morishita,Y.Togami and K.Tsushima, J.Phys.Soc. Japan 54, 37 (1985).

[2] N.Sato, J.App1.Phys. 59,2514(1986) .

[3] S. Honda, M.Ohkoshi and T.Kusuda, IEEE Trans. Magn. MAG-22, 1221 (1986).

[4] K. Yoden, N.Hosoi to, K. Kawaguchi ,K.Mibu and T.Shinjo, Jpn.J.Appl.Phys. 27, 1680(1988) .

[5]S.Honda, S.Nishimura, M.Ohtsu and T.Kusuda, Res.Meeting of Magnetics,MAG-88-198,9(1988).

[6] S. Honda, S.Nishimura and T.Kusuda, IEEE Trans.Magn. 25,4027 (1989).

[7]D.J.Se11myer and Z.S.Shan, MRS Int'1.Mtg.Adv.Mats. 10,269(1989).

[8] Z.S.Shan and D.J.Sellmyer, J.Appl. Phys. 67,5713(1990).

[9] Y. Mimura and $\mathrm{N}$. Imamura, Appl . Phys. Lett . 28, 746(1976).

[10]N.Heiman, K.Lee,R.I.Potter and S.Kirkpatrick, J.App1.Phys .47, 2634(1976). 